\title{
Application of Business-Driven Decision Making to RESTful Business Processes
}

\author{
Qinghua $\mathrm{Lu}^{2,1}$, Xiwei $\mathrm{Xu}^{1,2}$, Vladimir Tosic ${ }^{1,2}$, and Liming $\mathrm{Zhu}{ }^{1,2}$ \\ ${ }^{1}$ NICTA, Australian Technology Park, Sydney, NSW, Australia \\ ${ }^{2}$ University of New South Wales, Sydney, NSW, Australia \\ \{Qinghua. Lu, Xiwei.Xu, Vladimir. Tosic, Liming. Zhu\} @nicta.com. au
}

\begin{abstract}
Runtime adaptability is a desired quality attribute in business processes, particularly cross-organizational ones. Past work showed that designing and implementing business processes following the REpresentational State Transfer (REST) principles increases runtime adaptability. However, the past solutions for RESTful business processes (RESTfulBP) were limited to manual selection of process fragments to be composed at runtime. Therefore, we have now integrated into the RESTfulBP system an extended version of our MiniZnMASC middleware to enable concurrent selection of different RESTfulBP process fragments for different classes of user at runtime. This selection maximizes overall business value, while satisfying all given constraints. We also extended the RESTfulBP runtime engine with a process fragment processor, a constraint processor, a process fragment repository, and several types of monitoring resources. Experiments with prototype implementations showed that our solutions are feasible, functionally correct, business beneficial, with relatively low performance overhead, and with satisfactory scalability.
\end{abstract}

Keywords: REST, business-driven IT management, middleware, Web service composition management, decision support, middleware, autonomic computing.

\section{Introduction}

To improve adaptability of business processes, we previously designed an architecture style "RESTful business processes (RESTfulBP)" [1] that adapts the REpresentational State Transfer (REST) principles [2] to business process design, implementation and execution. Compared with the traditional business process using flow-based languages (e.g., BPEL), RESTfulBP has two significant characteristics: 1) at the process level, RESTfulBP models business processes in a declarative style at design time and allows reusable process fragments to be bundled, unbundled and re-bundled flexibly and rapidly at runtime; 2) RESTfulBP can fully utilize the mechanisms of the HTTP protocol to provide a lightweight adaptable infrastructure supporting business process execution and adaptation. In RESTfulBP, decision making points are where process fragments can be bundled, unbundled, and re-bundled. When several process fragments can be used at a decision making point, it is necessary to determine which one to use. Since the affected RESTfulBP users can have different characteristics, one 
single selection of a process fragment for all users can rarely achieve best business value for the RESTfulBP owner organization. It is often necessary to concurrently examine selection of process fragments for all affected users. In past work [1], this decision is made manually by a "knowledge worker", who is authorized to manage business process instances. However, it is hard for humans to have entire knowledge about the runtime RESTfulBP states and understand multifaceted interdependencies between diverse components and metrics at various levels of abstraction. It is also difficult for knowledge workers to manually calculate business value that each process fragment leads to and decide which process fragment to execute taking into account business strategies of the RESTfulBP owner organisation. Therefore, a business-driven decision making support tool is needed to assist knowledge workers in the process fragment selections at runtime.

Based on the past studies on autonomic business-driven IT management [3, 4], we previously designed MiniZnMASC $[5,6]$ that is an autonomic business-driven decision making middleware for adaptation of Web service compositions. In this paper, we extend MiniZnMASC with new algorithms and build it into RESTfulBP to provide runtime decision making support in the process fragment selections at each decision making point of RESTfulBP. The adapted version of MiniZnMASC first examines all available process fragments at each decision making point and selects different groups of process fragments for different classes of users, in ways that satisfy the business value constraints and other constraints (e.g., resource limitations). The use of MiniZnMASC can be fully automatic, when it selects and invokes the combination of process fragments (one process fragment per class of user) that maximizes business value. However, a more realistic use scenario is semi-automatic, when a knowledge worker examines MiniZnMASC advices and selects the final combination of process fragments based on own knowledge and experience. The new architecture of RESTfulBP with built-in MiniZnMASC introduces a process fragment processor, a constraint processor, a process fragment repository, and several kinds of monitoring resources for process fragment processing. The recorded monitored data are sent to MiniZnMASC in an XML file and are used in adaptation decision making. The adaptation actions decided by MiniZnMASC are invoked by sending a HTTP request.

The remainder of this paper is organized as follows. Section 2 introduces a motivating example for this work. Section 3 examines related work and summarizes our past work on RESTfulBP and MiniZnMASC. Section 4 discusses in detail how we integrated the extended MiniZnMASC into RESTfulBP. Section 5 evaluates the proposed solutions. The last section concludes the paper.

\section{Motivating Example}

The motivating example illustrating the usefulness of our solutions is the loan approval process from the Lending Industry XML Initiative (LIXI) [7]. It is a humanintensive process that largely depends on the knowledgeable human for guidance. Thus, the software is used to assist the human to direct the process rather than completely automate the process execution. As outlined in the upper part of Fig. 1, this is 
a complicated business process, where entities are from different businesses and entities of the same type can differ. Thus, the lending institution classifies its users into three classes according to their credit history and previous loan records: gold, silver, and bronze. It also offers different service levels, which have different guaranteed technical QoS, prices per invocation, and penalties if the guarantees are not met.
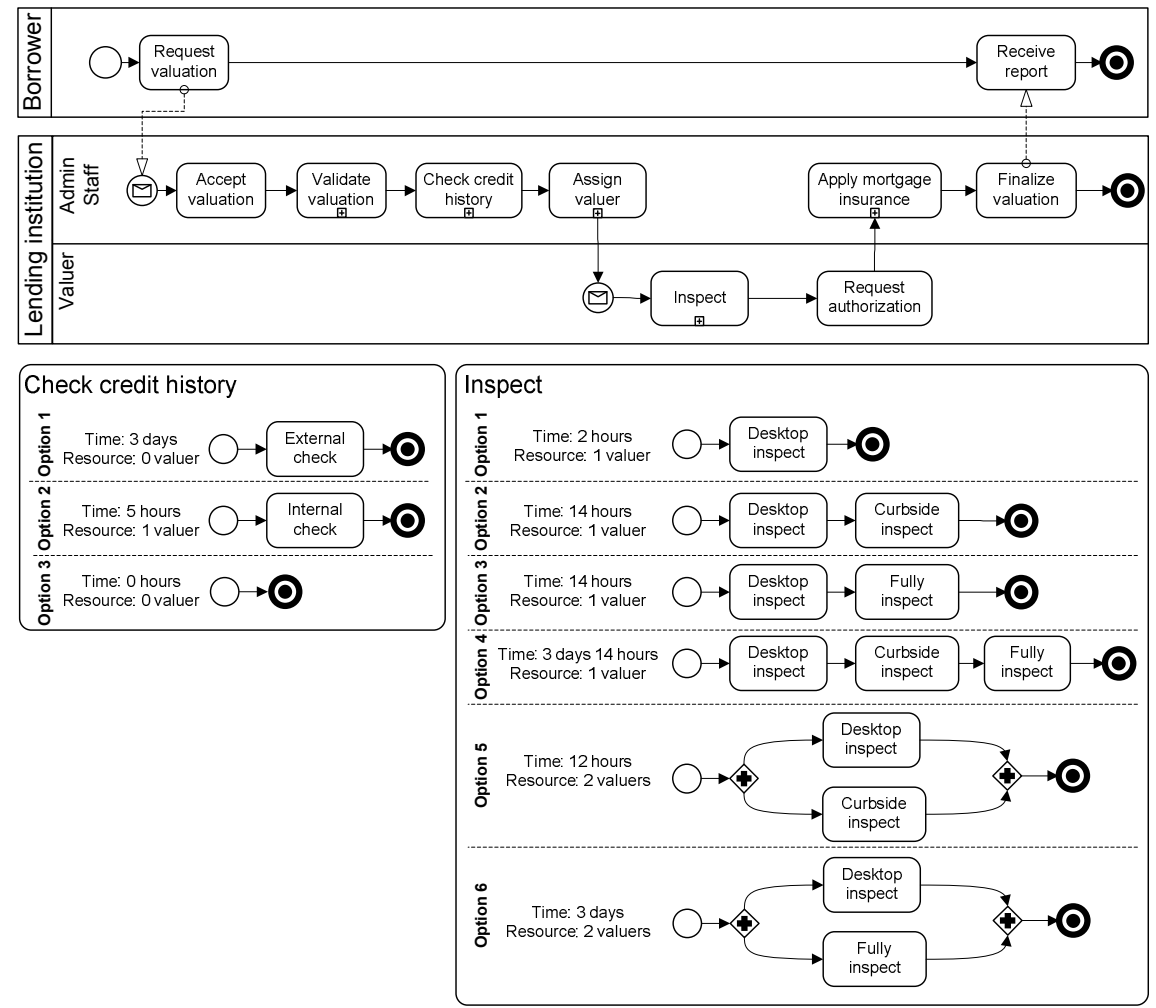

Fig. 1. A loan approval process with possible process fragments

There are many decision making points in the loan approval process where advanced decision making is necessary. Due to space limitations, we only discuss the decision making of the "Inspect" sub-process, for which the lower-right part of Fig. 1 outlines possible process fragments and corresponding time/resource constraints. (The lower-left part of Fig. 1 shows another sub-process, "Check credit history", as an illustration that there are many decision making points, but we do not have space to describe this sub-process here.) There are three types of property inspection: desk-top, curbside, and full. Desk-top inspection estimates the property value based on the existing data repository - it has a negligible cost and can be completed by 1 valuer within 2 hours. Curbside inspection includes the outside inspection (without entering the property) and requires 0.5 day for 1 valuer to complete. Full inspection includes both inside and outside inspections and may take 3 days or more to be completed, because 
the valuer has to make an appointment with the property owner. The possible process fragments at the "Inspect" decision making point are shown in the expanded "Inspect" sub-process. The initiation of the inspection is very flexible in terms of the number of the inspections and the sequence of the chosen inspections. MiniZnMASC filters the process fragments depending on the constraints and monitored data and sorts them based on business value. However, there are some knowledge and runtime situations that are hard to capture in predefined policies, so a knowledge worker might be required to make the final decision among the filtered process fragments.

For one example runtime situation at the "Inspect" sub-process, the Table 1 shows the number of currently running loan approval process instances in each class of user and the calculated business value and cost of each adaptation option per instance. Further, the lending institution has 6 valuers and the completion time limit for the "Inspection sub-process" is 4 days. Two other constraints limit the overall business value to at least $\$ 6000$ and the overall cost to at most $\$ 2000$. Domain experts provide information that helps determine business values, costs, and various constraints for decision making. The advices produced by MiniZnMASC are in the last line of Table 1.

Table 1. Business value (V) and cost (C) (AU\$) of different adaptation alternatives for different classes of user at the "Inspect" decision making point

\begin{tabular}{|c|c|c|c|}
\hline Class of user & Gold & Silver & Bronze \\
\hline Number of instances & 2 & 3 & 5 \\
\hline Option 1 & V: $500 ; C: 50$ & V: 300; C: 50 & V: 100; C: 50 \\
\hline Option 2 & V: 400; C: 100 & V: 600; C: 100 & V: 200; C: 100 \\
\hline Option 3 & V: 400; C: 150 & V: 400; C: 150 & V: 200; C: 150 \\
\hline Option 4 & V: 300; C: 200 & V: 400; C: 200 & V: 600; C: 200 \\
\hline Option 5 & V: 1000; C: 100 & V: 400; C: 100 & V: 200; C: 100 \\
\hline Option 6 & V: $800 ; C: 150$ & $\mathrm{~V}: 400 ; \mathrm{C}: 150$ & V: $200 ; C: 150$ \\
\hline Selected adaptation & Option 5 or Option 6 & Option 2 & Option 4 \\
\hline
\end{tabular}

Section 4 explains how our extended MiniZnMASC middleware makes such decisions based on policies specified in the WS-Policy4MASC language. We have used the described example for evaluation of our solution (cf. Section 5).

\section{Related Work and Background}

\subsection{Related Work}

Process fragment is not a new concept and it has been shown previously that that dividing business process into process fragments can improve the flexibility and dynamic evolution [1,8]. For example, the process fragment concept in our work is similar to a worklet from [8] - a small, self-contained, complete workflow process that handles one task in a larger, composite process. It can be also regarded as a 
process-level continuation in distributed business processes. A similar concept has been used in [9], but this approach is aimed to overcome the bottleneck of centralized process and improve the performance, while our work has significantly broader scope. A novelty of RESTfulBP is that it utilizes the existing Web infrastructure to provide a light-weight architecture for the execution of process fragments. RESTfulBP also inherits good characteristics of REST, such as loose coupling and interoperability. Although REST [2] and Resource-Oriented Architecture [10] principles are well established, their application to Web-based business process systems has not been well understood. Several proposals to bring REST to business processes have emerged from the industry and research community. Most of them extend SOA standards with RESTful interfaces $[10,11]$ or impose selected REST constraints on business process implementation [12-15]. However, these have several limitations, which RESTfulBP overcomes. Firstly, the methods for introducing additional constraints focus only on two constraints: uniform interfaces and "hypermedia as the engine of application state". They ignore other useful mechanisms of the Web infrastructure: content negotiation, rich metadata, and transfer of process fragments to enable truly distributed and localized process execution. There are very few style implementation guidelines and associated methodologies. Secondly, there is a lack of full support for workflow patterns. Finally, confusion arises when different and sometimes conflicting additional constraints are proposed without a clear definition of the new style and associated methodology for implementing it.

On the other hand, there are many publications on particular types of adaptation of business processes. We provided a classification and a relatively detailed analysis of many additional related works in [16]. While in industry practice adaptation decision making is still mainly done by human administrators, the vast majority of research focuses on adaptation with minimal help from humans. However, the past adaptation decision making algorithms predominantly choose adaptation that maximizes technical metrics (e.g., [17, 18]), while maximization of business metrics is still an open research area [4, 16]. While [19] is not directly on adaptation of business processes, some of its solutions could be reused in our context. It presented a system for maximization of business metrics that schedules the triggered management policies by minimizing the penalty specified in service level agreements (SLAs), but it did not examine resolution for conflicting policies, which is a critical problem in policy-based management. Different adaptation triggers often require different monitoring, decision making, and adaptation execution. For example, if a management system does not monitor business-related events, it will not be able to recognize the adaptation needs they cause, so it will not be able to react and adapt appropriately. However, the vast majority of past works (e.g., [17, 20]) researching adaptation of business processes focused on adaptations triggered by technical reasons, while only a few research projects (e.g., [21, 22]) examined the impact of business causes (but without addressing them completely). In particular, [21] identified three types of adaptation causes: exceptions, business policy changes, and business model changes. There are many other additional causes that were not addressed, such as infrastructure updates, business strategy changes, and business customer changes. Adaptation of a business process instance could change various aspects of system configuration and execution: 
structure of the implemented business process type (e.g., replacing one block of activities with another for all instances) [17], Web services used for the implementation (e.g., replacing a faulty service) [23], execution of the instance (e.g., rolling back to a checkpoint) [24], or contracts with customers (e.g., changing SLAs) [25]. There is a lot of research literature presenting support for individual types of change, but a comprehensive support for all these types of change was first provided in the WSPolicy4MASC language and the MASC middleware [23], which is the predecessor of the research presented in this paper. Our work is different from traditional solutions of optimization problems in business processes. We focus on the runtime decision making support for concurrent adaptation of multiple RESTful business process instances with consideration of business value for the process owner.

\subsection{Background}

\subsubsection{The RESTfulBP Architecture Style}

The RESTfulBP architectural style is a set of architectural constraints [1] that aims to establish communication and coordination mechanisms among participants in a business process from a peer-to-peer and distributed point of view. Small pieces of workflow logic, called "process fragments", are defined within each endpoint and can be transferred to other endpoints at runtime. RESTfulBP models four types of business process entities (process, instance, task, and state) as resources identified by declarative URIs [10]. The entity resources are manipulated through a set of uniform methods, resembling the standard HTTP methods (GET, PUT, POST, and DELETE). The process entity resources are connected through a Microformat [21]. The Microformat-based messages are used to communicate routing information at runtime. RESTfulBP uses content negotiation and authentication mechanisms provided by the Web infrastructure to choose an appropriate representation of an entity resource for the role-based requester. A server can estimate the desired representation format according to the ACCEPT request header, which indicates the preferred media type. The current media type is extended to indicate the role of the requesting participant. RESTfulBP communicates exception information in the message header. The HTTP protocol is extended with an optional header field "Exception" to denote the type of exception, so that the requestor can get sufficient information from the message header only. The tool support for RESTfulBP includes: an annotation tool based on the Eclipse project BPMN Modeler [22], a programming API that facilitates implementation of RESTful process-aware systems, and a runtime engine that powers the programming API.

\subsubsection{The MiniZnMASC Middleware}

In complex long-running business processes, changes happen often (e.g., business goal changes, service performance changes). When such changes occur during runtime, the affected Web service compositions should be adapted. This adaptation can be usually done in several ways and advanced decision making is needed to determine how to proceed. MiniZnMASC [5] is a runtime autonomic decision making middleware for adaptation of Web service compositions. It implements novel 
decision-making algorithms that can concurrently make different adaptation decisions for different classes of instance in a way that achieves maximum overall business value while satisfying all given constraints. A "class of instance" is a group of Web service composition instances that share a combination of characteristics that warrants adaptation in the same way. The most important characteristics are: the implemented business process type, the executed Web service composition, the current position/state within the running Web service composition, and the class of consumer. The decision-making algorithms in MiniZnMASC use information specified as policy assertions in WS-Policy4MASC. WS-Policy4MASC $[4,23]$ is a policy language that can describe various adaptations and all information necessary for decision making. WSPolicy4MASC extends the WS-Policy industry standard and defines five new types of policy assertions: 1) goal policy assertions (GPA) prescribe conditions to be met (e.g., desired response time), 2) action policy assertions (APA) list adaptation actions to be performed in particular situations, 3) utility policy assertions (UPA) specify business values for particular situations, 4) probability policy assertions (PPA) specify probabilities of occurrence, and 5) meta policy assertions (MPA) describe how to select among alternative adaptation decisions to maximize business value.

\section{Extending MiniZnMASC and Integrating It into RESTfulBP}

To provide business-driven decision making support for the runtime RESTfulBP process fragment selection, we extend the MiniZnMASC middleware with new decision making algorithms. The new decision making algorithms concurrently select different combinations of process fragments (addressing all classes of user) at a decision making point. The selections found by MiniZnMASC satisfy all given business value (e.g., cost) constraints and other constraints (e.g., resource limitations) and depend on business metrics and business strategies, plus operational conditions (e.g., current number of users in each class). The selected combinations of process fragments can be provided as an advice for knowledge workers who make final decisions. Alternatively, when knowledge workers completely trust the specified WSPolicy4MASC policies, MiniZnMASC can automatically select and invoke the combinations that it determines optimal from the business viewpoint, similarly to $[4,5]$.

While these algorithms are based on the autonomic MiniZnMASC algorithms described in $[4,5]$, the main differences are in the constraint programming model: 1) the new model can select more than one process fragments for each class of RESTfulBP user while the original model can only select one adaptation action for each class of Web service composition instance; 2) the new model handles various constraints including business value constraints (e.g., discarding a process fragment that leads to business value smaller than the minimal acceptable one), time constraints, and resource constraints, while the original model had a business value optimisation objective and primarily worked with cost constraints. The new constraint programming model makes MiniZnMASC more suitable for integration into RESTfulBP as a decision making support tool. The novel integration of MiniZnMASC with RESTfulBP enables semi-automatic business-driven adaptations in complex, multi-user RESTfulBP with minimal help from human knowledge workers. 
There were four challenges to integrate MiniZnMASC into RESTfulBP: 1) tailor the previous automatic decision making algorithms to semi-automatic decision making algorithm and tailor the previous modelling in MiniZnMASC to describe the selection of process fragments in RESTfulBP; 2) design external monitoring mechanism in RESTfulBP that can send real-time data to MiniZnMASC; 3) externally execute the adaptation actions; 4) design the overall architecture of the integrated system.

\subsection{The Constraint Programming Model for Decision Making Algorithms}

In this subsection, we describe our solution to the first challenge identified above, while our solutions to the other three challenges are presented in the next subsection. We represent our decision making model in constraint programming and code it in the constraint programming language MiniZinc [25]. The Policy Conflict Resolution module (cf. Fig. 2) of MiniZnMASC contains the MiniZinc solver, which instantiates the model with values from WS-Policy4MASC files and runtime monitoring data and solves this instantiated model for the new MiniZnMASC optimization algorithm.

We use the following notation. Unless noted otherwise, the listed variables are specified in WS-Policy4MASC files. $N$ is the number of classes of user. $X_{n}(n=1, \ldots, N)$ is the current number of instances in class $n$ and it is determined from runtime monitoring data. $M_{n}(n=1, \ldots, N)$ is the number of process fragment options (at the examined decision point) for a user in class $n . A_{n, i}\left(n=1, \ldots, N ; i=1, \ldots, M_{n}\right)$ is the $i$-th process fragment option for class $n$. $K$ is the number of business value types [4] that can be reasoned about. $V_{k, n, i}\left(k=1, \ldots, K ; n=1, \ldots, N ; i=1, \ldots, M_{n}\right)$ is the summary business value of type $k$ for process fragment option $A_{n, i}$. It is calculated by our algorithm, summarized in [4], for calculation of summary business metrics from the values in WS-Policy4MASC utility and probability policy assertions. (The probabilities specify uncertainties and risks.) The algorithm is implemented in MiniZnMASC as Java code, so in this constraint programming model we just use its result $V_{k, n, i}$. We also derived a precise mathematical formula showing how $V_{k, n, i}$ is calculated, but this is not crucial for this paper. $W_{k}(k=1, \ldots, K)$ is the weight of business value type $k$. These weights are specified in WS-Policy4MASC meta policy assertions and are usually in the interval $[-1,1]$ (negative values are for costs).

The summary business value of all used business value types for a process fragment option $A_{n, i}$ is calculated as: $B_{n, A_{n, i}}=\sum_{k \in U s e d B V T s} W_{k} * V_{k, n, i}(k=1, \ldots, \quad K$; $n=1, \ldots, N ; i=1, \ldots, M_{n}$ ). The set UsedBVTs contains all business value types deemed relevant (as specified in the used WS-Policy4MASC meta policy assertion) for comparing business worth of process fragment options. This feature addresses the fact that in different business situations different sets of business value types are relevant.

The problem of finding the globally optimal set of process fragments can be represented in constraint programming as the task to find the set of $N$ process fragments $J_{n} \in\left\{A_{n, 1}, \ldots, A_{n, M_{n}}\right\}(n=1, \ldots, N)$ that satisfy all given constraints and have the highest summary business value. We use $j_{n}$ to denote $i$ of the chosen $A_{n, i}$, that is $J_{n}=A_{n, i} \Leftrightarrow j_{n}=i$. The most important are business value constraints describing business situations when a process fragment option should be discarded because it 


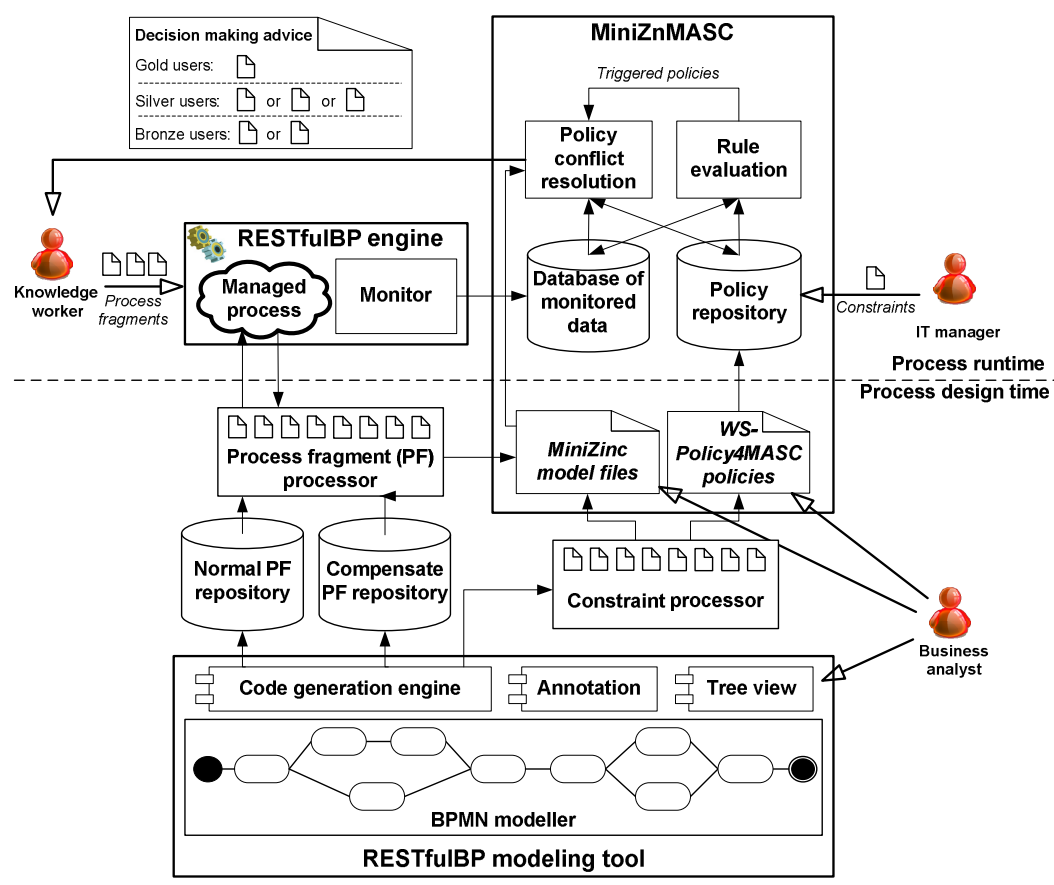

Fig. 2. Architecture of RESTfulBP with integrated MiniZnMASC

leads to business value that is smaller than the acceptable minimum. These constraints are modeled as: $\sum_{n=1}^{N} X_{n} * B_{n, j_{n}} \geq$ MinBusinessValue. Cost constraints describe common business situations when a process fragment option should be discarded because its short-term costs are higher than available funds. They are modeled similarly to: $\left|\sum_{n=1}^{N}\left(X_{n} * \sum_{k \in \text { CostBVTs }} W_{k} * V_{k, n, j_{n}}\right)\right| \leq$ CostLimit. Here, the set CostBVTs contains all business value types representing relevant costs (as specified in the used WSPolicy4MASC meta policy assertion). Resource constrains are another constraint type important in practice. Resources (e.g., memory, processor time, bandwidth, energy, etc.) are often limited and this is one of the main reasons why decisions of concurrent instances should be considered together instead of separately. WS-Policy4MASC enables definition of non-financial business value types that represent resource usage and use appropriate units. For example, it is possible to define that all non-financial costs with attribute Cause = "Memory" represent memory usage and have unit "GB". Then, a memory limit can be modelled similarly to: $\mid \sum_{n=1}^{N}\left(X_{n} * \sum_{k \in \text { MemoryBVTs }} W_{k} *\right.$ $\left.V_{k, n, j_{n}}\right) \mid \leq$ MemoryLimit, where the set MemoryBVTs contains all business value types that represent memory usage. Time constraints are yet another important constraint type, because time (both calendar time for completion of a task/sub-process and time that can be spent actively working on a task/sub-process) is often limited in business processes. Time constraints often depend on characteristics of a business process instance (e.g., gold class usually has lower time limits than bronze class). $P_{n, i}\left(n=1, \ldots, N ; i=1, \ldots, M_{n}\right)$ is the number of tasks/sub-processes in $A_{n, i}$ and $Y_{n, i, p} \quad(n=1, \ldots, \quad N ; \quad i=1, \ldots$, $\left.M_{n} ; p=1, \ldots, P_{n, i}\right)$ is a particular task/sub-process in $A_{n, i}$. Thus, time constraints 
can be modeled similarly to: $\operatorname{WorkTime}\left(Y_{n, i, p}\right) \leq \operatorname{WorkTimeLimit}\left(Y_{n, i, p}\right)$ and CalendarTime $\left(Y_{n, i, p}\right) \leq$ CalendarTimeLimit $\left(Y_{n, i, p}\right)$. WS-Policy4MASC and MiniZnMASC can also support additional constraint types, but they are not very common in practice.

\subsection{Architecture of RESTfulBP That Adds the Extended MiniZnMASC}

Fig. 2 shows the architecture of RESTfulBP with integrated MiniZnMASC middleware. The dash line in the middle separates the components at design time and runtime in perspective of the produced business process. At design time, the business process is defined by business analysts using BPMN with RESTfulBP annotations. The purpose of modeling is to identify the tasks in the business process at different levels of abstraction, the roles of the process participants, the control dependencies between the tasks (e.g., sequencing, parallel), and various constraints. Besides, the constraint programming models and management policies which provide the basis for MiniZnMASC are also defined by the business analyst according to all relevant RESTfulBP models and other business information, e.g., process fragments, constraints, business strategies, and operational conditions. At runtime, the RESTfulBP engine is used to execute the produced processes. RESTfulBP is designed for the human-intensive processes. Thus, a knowledge worker is usually required to drive the process execution, and MiniZnMASC is used to assist the knowledge worker in making more suitable decisions from a group of available process fragments. (As mentioned above, MiniZnMASC can make decisions without human involvement, but humans have to fully trust them and this might not be realistic in complex situations.)

\subsubsection{Process Design Time}

The RESTfulBP modeling tool is built on the basis of the Eclipse platform and several existing Eclipse projects. BPMN modeler is an Eclipse-based business process diagram editor. It realizes Business Process Modeling Notation (BPMN) [24] specification. The Annotation plug-in is implemented on top of the BPMN modeler by utilizing the extension mechanisms of Eclipse. It builds upon several extension points provided by the BPMN modeler and the Eclipse platform. The Annotation plug-in allows the developers to annotate various elements of BPMN diagrams with RESTfulBP information, which is essential for the Code Generation Engine to generate code. The Code Generation Engine can extract useful information from the diagram and generate process skeletons and constraints for certain tasks or sub-process. It consists of four modules: workflow pattern detector, constraints detector, code generation wizard, and code generation template. The workflow pattern detector can recognize which workflow pattern a certain task belongs to, by referring to its pre-and-post tasks. The identified workflow pattern is used by the code generation template to create tasks and process fragments. Developers can configure the generated project using the code generation wizard, e.g. by choosing the path of the dependent library. Then, the code generation engine combines the information from both the template and the wizard forms to create an executable process. The process fragments extracted from the normal process are the normal process fragments, while the process fragments extracted from the compensation process are the compensate process fragments used for exception handling. The information is stored in an XML file as an input of the Process Fragment Processor. The Process Fragment Processor extracts the identification number of each process fragment and stores it for different decision making 
points and possible exception points. The output of the process fragment processor includes some of the information required by MiniZinc models, e.g. the number of available process fragments. The constraints detector can extract the constraints for the tasks. The information is stored in an XML file as an input of the Constraint Processor. The Constraint Processor treats all received constraints and stores them for different constraint types. The Tree Viewer organizes the tasks and corresponding process fragments into a tree construct. All tasks are children of the root node. A task can contain an arbitrary number of process fragments as its children.

\subsubsection{Process Runtime}

During process execution, RESTfulBP provides a runtime environment that allows process fragments to be exchanged between process participants. The process is driven by a knowledge worker, who is responsible for selecting (based on own knowledge) the most suitable process fragment to execute from a group of process fragments. MiniZnMASC can assist the knowledge worker by selecting for different classes of user smaller groups of process suitable fragments that satisfy given constraints. The selections made by MiniZnMASC are based on monitored data from the runtime execution and according to policies in WSPolicy4MASC [4, 23]. If changes happen in the operational environment, IT managers may update the constraints and relevant policies in the Policy Repository at runtime. MiniZnMASC is used not only to assist the knowledge worker to make decisions in normal process execution, but also to provide an exception handling mechanism (e.g., when a server is down).

For process monitoring, the Monitor module of RESTfulBP uses a set of monitoring resources to provide monitoring functionality. The monitoring resource on the top-level is addressed by a URI, e.g. " $\{$ processURI\}/monitoring/". It has a set of subresources, each of which represents a type of runtime data to be monitored. For example, "\{processURI\}/monitoring/responsetime" represents the response time metric, while " $\{$ processURI $\} /$ monitoring/availability" represents the availability metric. These QoS metrics can be used to measure different service levels of process entities, which are also represented by sub-resources of QoS metric resources. For example, response time of a certain process instance can be accessed trough the resource " $\{$ processURI\}/monitoring/responsetime/\{instanceId $\} "$. Similarly, response time of a certain task of the same process instance is represented by "\{processU$\mathrm{RI}\} /$ monitoring/responsetime/ $/$ instanceId $\} /\{$ taskId $\} "$. The monitoring resources record all relevant runtime data automatically at runtime. The monitored data is stored in the Database of Monitored Data, which includes both technical metric data (e.g., measured response time) and business metric data (e.g., paid prices and penalties).

\section{Evaluation}

\subsection{Feasibility, Functional Correctness, and Business Benefits}

We implemented prototypes of the extended MiniZnMASC middleware and the new RESTfulBP system containing MiniZnMASC and performed with it a number of diverse tests, using the motivating example from Section 2 and other examples. The new MiniZnMASC prototype was built by extending the prototype discussed in [5], so it was also implemented in Java and uses the PostgreSQL database. The new prototypes show that implementation of our solutions is feasible. 
We first tested functional correctness of the MiniZnMASC by comparing results calculated by the prototypes and by hand, in various scenarios. Since we found no differences, these results indicate that the new algorithms do not have functional errors. We then comprehensively tested the new overall RESTfulBP system that includes the extended MiniZnMASC. After debugging minor errors, we found no further problems with the prototype implementation of our core solutions.

For the motivating example we also calculated summary business value (incl. possible revenue and relevant costs) for different possible decisions, some of which are shown in Table 2. These results confirm that using the new MiniZnMASC algorithms for automatic selection of process fragments to be executed leads to business benefits. The combination of these tests of functional correctness and business benefit shows that the developed algorithms and middleware will select process fragments best from the business viewpoint, according to the information specified in policies.

Table 2. Total business value (V) and cost $(\mathrm{C})$ is AU\$ of different decisions

\begin{tabular}{l|l}
\hline Decision & Business benefits \\
\hline \hline Single Selection of Option 1 & V: $2400 ;$ C:500 \\
Single Selection of Option 2 & V: $3600 ;$ C: 1000 \\
Single Selection of Option 3 & V: $3000 ;$ C: 1500 \\
Single Selection of Option 4 & V: $4800 ;$ C: 2000 \\
Single Selection of Option 5 & V: $4200 ;$ C: 1000 \\
Single Selection of Option 6 & V: $3800 ;$ C: 1500 \\
Selections by MiniZnMASC & V: $6400 \sim 6800 ;$ C: $1500 \sim 1600$ \\
\hline
\end{tabular}

\subsection{Performance and Scalability}

For the evaluation of performance and scalability, we used a Hewlett-Packard laptop model HP EliteBook6930p with Intel Core 2 Duo CPU T900 2.53GHz processor and $4.00 \mathrm{~GB}$ of RAM memory, running 32-bit Windows Vista operating system. This configuration is near the lower end of environments in which the RESTfulBP system with MiniZnMASC might be run in practice. The fact that we found performance satisfactory in this low-end environment gives us confidence that performance will not be a problem in practical use of our solutions. To minimize the impact of noise (e.g., background operating system processes that we were not able to switch off), we repeated tests hundreds of times at different times of day and averaged their results.

Table 3. Performance of decision making (DM) and conflict resolution algorithm (CRA), with increasing number of conflicting action policy assertions (APAs) and utility policy assertions (UPAs)

\begin{tabular}{l||l|l}
\hline Test case & Execution time of DM & Execution time of CRA \\
\hline \hline 3 APAs, each with 2 UPAs & Average: $250 \mathrm{~ms}$ & Average: $16 \mathrm{~ms}$ \\
& Range: $234-266 \mathrm{~ms}$ & Range: $15-32 \mathrm{~ms}$ \\
\hline 10 APAs, each with 32 UPAs & Average: $905 \mathrm{~ms}$ & Average: $31 \mathrm{~ms}$ \\
& Range: $889-936 \mathrm{~ms}$ & Range: $16-32 \mathrm{~ms}$ \\
\hline \multirow{2}{*}{100 APAs, each with 64 UPAs } & Average: $12309 \mathrm{~ms}$ & Average: $140 \mathrm{~ms}$ \\
& Range: $12262-12449 \mathrm{~ms}$ & Range: $140-156 \mathrm{~ms}$ \\
\hline
\end{tabular}


In one set of performance and scalability tests, we increased the complexity of decision making in a decision point. We simultaneously increased two aspects of this complexity: 1) the number of available process fragments (represented through conflicting WS-Policy4MASC action policy assertions - APAs); 2) the complexity of calculating summary business value for each process fragment (represented through the number of WS-Policy4MASC utility policy assertions - UPAs). Using the Java "System.currentTimeMillis()" call, we measured the execution time of whole MiniZnMASC business-driven decision making (DM) and the execution time of only the Confliction Resolution Algorithm (CRA) that is core to this decision making. Table 3 shows the measured results of the range and average of the execution time for some test scenarios. The overall execution time of decision making in MiniZnMASC rises because the execution time of the summation of business values for each conflicting action policy assertion increases with increasing number of conflicting action policy assertions and utility policy assertions. The last test case (100 conflicting action policy assertions, 64 utility policy assertions) is much more complicated than realistic scenarios in practice, so $12.3 \mathrm{sec}$ is not an issue. It is important to note that in realistic application scenarios of MiniZnMASC to RESTfulBP the number of conflicting action policy assertions will be low, while the overall number of action policy assertions can be huge. We also checked that the number of additional non-conflicting action policy assertions in the MiniZnMASC Policy Repository has no significant effect on performance, even when there are hundreds of action policy assertions.

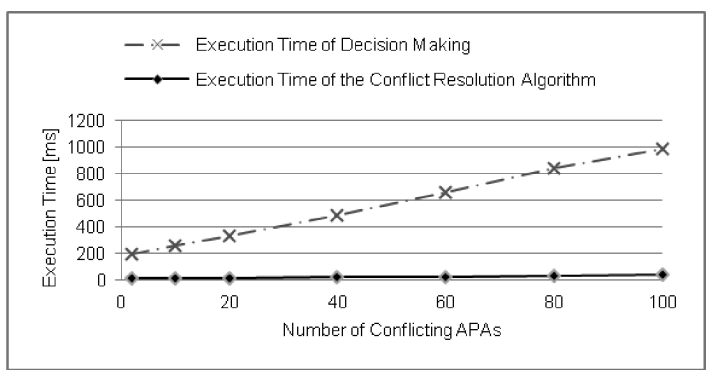

Fig. 3. Performance results with increasing number of conflicting action policy assertions (APAs), each with 2 utility policy assertions (UPAs)

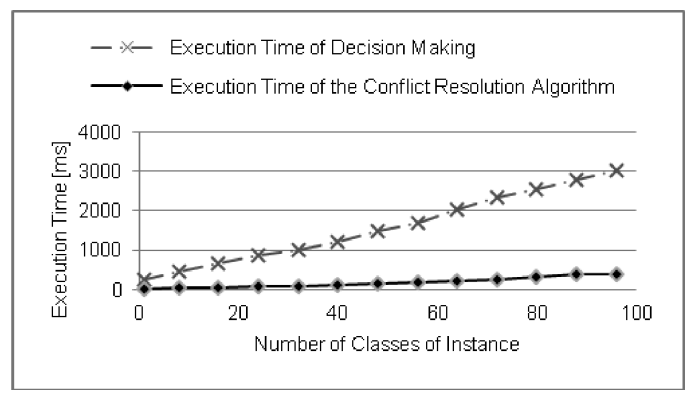

Fig. 4. Performance results with increasing number of classes of instance 
Fig. 3 shows the performance measurement results when we increased the number of action policy assertions, while Fig. 4 shows the performance measurement results when we increased the number of classes of user. In both figures, the upper line is for the execution time of the overall MiniZnMASC business-driven decision making, while the lower line is for the execution time of the Conflict Resolution Algorithm. Both lines show linearity, which is good. In our application of MiniZnMASC to RESTfulBP, the decision making component first decides the action policy assertions triggered by events received from the external monitoring modules and finds utility policy assertions and probability policy assertions for the relevant action policy assertions. Then, if the number of action policy assertions is more than one, the new MiniZnMASC integrated into RESTfulBP runs the Conflict Resolution Algorithm to select among the conflicting action policy assertions. The results in Fig. 4 show that the majority of the execution time of the overall decision making is spent on deciding triggered action policy assertions and setting utility policy assertions and probability policy assertions, and not on the Conflict Resolution Algorithm.

\section{Conclusion}

The past solution for RESTfulBP was limited to manual selection of process fragments to be composed at runtime. Therefore, in this paper, we extended our MiniZnMASC middleware with new decision making algorithms to enable semi-automatic concurrent selection of different RESTfulBP process fragments for different classes of user at runtime. The selections suggested by MiniZnMASC take into consideration business metrics, business strategies, and operational conditions (e.g., the current number of users in each class). These selections maximize overall business value while satisfying all given constraints. The new algorithms required several extensions of the constraint programming models and the MiniZnMASC middleware.

We also proposed new architecture of RESTfulBP that integrates MiniZnMASC middleware. In particular, we introduced a process fragment processor, a constraint processor, a process fragment repository, and several types of monitoring resources into the RESTfulBP runtime engine to automatically record values of different technical and business metrics. The monitored runtime data is passed to MiniZnMASC in an XML file, which is used in the decision making. MiniZnMASC results are usually provided as a decision making advice for knowledge workers who make final decisions (although it is also possible for MiniZnMASC to select and invoke process fragments without human intervention). New prototypes were implemented using Java, PostgreSQL database, and the MiniZinc solver. Experiments with the prototypes showed that our solutions are feasible, functionally correct, business beneficial, with relatively low performance overhead, and with satisfactory scalability.

Acknowledgments. NICTA is funded by the Australian Government as represented by the Department of Broadband, Communications and the Digital Economy and the Australian Research Council through the ICT Centre of Excellence program. 


\section{References}

1. Xu, X., Zhu, L., Kannengiesser, U., Liu, Y.: An Architectural Style for Process-Intensive Web Information Systems. In: Chen, L., Triantafillou, P., Suel, T. (eds.) WISE 2010. LNCS, vol. 6488, pp. 534-547. Springer, Heidelberg (2010)

2. Fielding, R.: Architectural Styles and the Design of Network-based Software Architectures. Universityof California, Irvine (2000)

3. Bartolini, C., Sahai, A., Sauve, J.P.: Proceedings of the Second IEEE/IFIP Workshop on Business-Driven IT Management. IEEE (2007)

4. Tosic, V.: Autonomic Business-Driven Dynamic Adaptation of Service-Oriented Systems and the WS-Policy4MASC Support for Such Adaptation. Intl. J. of Systems and ServiceOriented Eng. (IJSSOE) 1, 79-95 (2010)

5. Lu, Q., Tosic, V.: Support for Concurrent Adaptation of Multiple Web Service Compositions to Maximize Business Metrics. In: Proc. of IM 2011, pp. 241-248. IEEE (2011)

6. Lu, Q., Tosic, V., Bannerman, P.L.: Support for the Business Motivation Model in the WS-Policy4MASC Language and MiniZnMASC Middleware. In: Kappel, G., Maamar, Z., Motahari-Nezhad, H.R. (eds.) ICSOC 2011. LNCS, vol. 7084, pp. 265-279. Springer, Heidelberg (2011)

7. LIXI (Lending Industry XML Initiative). Web resource, http: / / www . Iixi . org . au/

8. Adams, M., ter Hofstede, A.H.M., Edmond, D., van der Aalst, W.M.P.: Worklets: A Service-Oriented Implementation of Dynamic Flexibility in Workflows. In: Meersman, R., Tari, Z. (eds.) OTM 2006. LNCS, vol. 4275, pp. 291-308. Springer, Heidelberg (2006)

9. Yu, W.: Consistent and Decentralized Orchestration of BPEL Processes. In: Proc. of SAC 2009, pp. 1583-1584. ACM (2009)

10. Richardson, L., Ruby, S.: RESTful Web Services. O'Reilly Media (2007)

11. Overdick, H.: Towards Resource-Oriented BPEL. In: Emerging Web Services Technology, vol. II, pp. 129-140. Springer (2008)

12. Pautasso, C.: BPEL for REST. In: Dumas, M., Reichert, M., Shan, M.-C. (eds.) BPM 2008. LNCS, vol. 5240, pp. 278-293. Springer, Heidelberg (2008)

13. Webber, J., Parastatidis, S., Robinson, I.: How to GET a Cup of Coffee. Web resource, http: //www. infoq.com/articles/webber-rest-workflow

14. zur Muehlen, M., Nickerson, J., Swenson, K.: Developing Web Services Choreography Standards-The case of REST vs. SOAP. Decision Support Systems 40, 9-29 (2005)

15. Rest-client. Web resource, http://github. com/caelum/rest-client

16. Baker, M.: Hypermedia Workflow. Web resource, http: / /www . markbaker.ca/2002/12 / HypermediaWorkflow/

17. Chafle, G., Dasgupta, K., Kumar, A., Mittal, S., Srivastava, B.: Adaptation in Web Service Composition and Execution. In: Proc. ICWS 2006, pp. 549-557. IEEE (2006)

18. Tong, H., Zhang, S.: A Fuzzy Multi-attribute Decision making Algorithm for Web Services Selection Based on QoS. In: Proce. of APSCC 2006, pp. 51-57. IEEE (2006)

19. Lu, Q., Tosic, V.: MiniMASC: A Framework for Diverse Autonomic Adaptations of Web Service Compositions. In: Proc. of UIC/ATC (Worksh. ANS) 2010, pp. 460-468. IEEE (2010)

20. Aib, I., Boutaba, R.: Business-Driven Optimization of Policy-Based Management Solutions. In: Proc. of IM 2007, pp. 254-263. IEEE (2007)

21. Microformat. Web resource, http://microformats.org/

22. BPMN Modeler. Web resource, http://www. eclipse.org/bpmn/ 
23. Tosic, V., Erradi, A., Maheshwari, P.: WS-Policy4MASC - A WS-Policy Extension Used in the Manageable and Adaptable Service Compositions (MASC) Middleware. In: Proc. of ICWS 2007, pp. 458-465. IEEE (2007)

24. Business Process Model and Notation (BPMN) 1.1. Web resource, http: / / www . omg. org/spec/BPMN/1.1/

25. Nethercote, N., Stuckey, P.J., Becket, R., Brand, S., Duck, G.J., Tack, G.: MiniZinc: Towards a Standard CP Modelling Language. In: Bessière, C. (ed.) CP 2007. LNCS, vol. 4741, pp. 529-543. Springer, Heidelberg (2007) 